\title{
Gesture-based control of the 3D visual representation of document collections for exploration and search
}

\author{
Angélica de Antonio*, Cristian Moral, Daniel Klepel and Martín J. Abente \\ Facultad de Informática, Universidad Politécnica de Madrid, Madrid, Spain \\ E-mails: \{angelica, cmoral,dklepel\}@fi.upm.es, mabente@alumnos.upm.es
}

\begin{abstract}
This paper describes an approach towards the interaction with 3D representations of large document collections. The goal was to provide the user with a highly dynamic environment in which even the very mapping strategy to position documents in space can be adjusted by the user depending on the specific task at hand, on his preferences, or on the context. A modification to the FDP algorithm is proposed, as well as a new gesture-based interaction paradigm in which the user can explore and search information in the collection just by simple hand movements. An experimental user evaluation was conducted to investigate the impact of the proposed approach on the precision of the mental model built by users through exploration, on the effectiveness in information search tasks, and on the general user satisfaction and perception of utility.
\end{abstract}

Keywords: 3D visualization and navigation, 3D interaction, information retrieval, force-directed placement, clustering, $k$-means

\section{Introduction}

Exploration and search of information contained in large document collections is increasingly a need, but many times also a problem. In fact, Internet offers such a huge quantity of information that it is in practice impossible to explore it exhaustively so as to fill a specific information need. In the last 30 years, many techniques have been proposed to visualize document collections. Some of them were developed for 3D virtual environments, as the third dimension increases the information that can be shown to the user. The application of one or another technique often depends on the type of documents to be visualized. For structured documents, specific visualizations highlight the structure and relations between data $[2,8,19,21]$, while for documents without any pre-defined internal structure and no metadata associated to them only the content can be analyzed in order to infer as much information as possible. In the last case, visualization techniques are more general and sophisticated, and then more complex [20].

This paper focuses on 3D visualization of non-structured document collections. The visual metaphor chosen, a sphere, is a simple and generic one in order to avoid overloading the visualization. Then, a collection of documents in the virtual environment is represented as a cloud of spheres. But visualization is definitely not enough to satisfy the user needs; interaction with the 3D representation is the key aspect to consider if the goal is to facilitate the user tasks.

The remainder of this document is divided into 4 sections. In Section 2 we detail the general process that must be followed to visualize a document collection in a 3D virtual environment, and we explain

\footnotetext{
*Corresponding author. E-mail: angelica@ fi.upm.es.
} 
the decisions we made in the implementation of every step. Section 3 deals with the interaction techniques we have proposed to allow the user exploring and extracting information from the 3D virtual environment. To evaluate the benefits and drawbacks of our proposal, we carried out an experiment, whose details and results are presented in Section 4. Finally, Section 5 reports the conclusions we have extracted from our work and proposes future research.

\section{3D visualization of document collections}

Based on the model described in [4], the information visualization pipeline can be split into four major stages. The first one consists in gathering, cleaning and preprocessing the documents, aiming at extracting from them as many attributes of interest containing relevant information as possible. Selecting these attributes requires a deep understanding of the task that should be carried on with the data and the nature of the data itself. The second stage consists in mapping visual characteristics to each of these attributes. The most common visual characteristics are color, shape, spatial position and movement [14], and they must be selected carefully in order to exploit the natural perception abilities of the users and considering the nature of each attribute [24]. The third stage consists in the generation of a visual representation of the scene making an efficient use of the screen space while keeping the cognitive load as low as possible. The fourth step deals with the interaction between the users and the virtual representation. Interaction is a fundamental issue regarding the design of any information visualization tool. Interaction mechanisms must be designed in order to assist the user's tasks, and they can act upon any of the previous stages including: select and manipulate a dataset, navigate through the environment and perform actions affecting the data or the system itself.

\subsection{Document preprocessing}

In our system, preprocess aims at reducing as much as possible the noise (words that have little or low semantic load), extracting the main concepts of each document and determining the similarity between any pair of documents in the collection. Many complementary treatments have been proposed to undertake this task: normalization, stopwords removal, Stemming [6,12,16,17], Part-Of-Speech Tagging $[1,3,18,23]$, Named Entity Recognition [9,15,26], and many others. Which of them to use depends on the efficiency and precision required in the retrieval of information. In our case, we look for a dynamic and interactive, and hence efficient, system, even at the expense of precision.

Our preprocessing phase consists of two main parts: the lexical analysis of each document, and clustering the documents into semantic groups based on the distance measure between them. The first part allows us to reduce the semantic noise contained in the documents and then to translate them into data structures treatable by computers. The process starts with the tokenization of the documents, this is extracting the individual words from them. In order to reduce the vocabulary as much as possible without loosing meaningful content, some filters are applied to the obtained tokens. These filters aim at homogenizing the tokens by normalizing them, for example by clearing accents, punctuation, email addresses, URLs, and numbers, among others. All filters are applied to individual tokens, as we use a bag-of-words approach, where context and order are not taken into account. Even if this causes the loss of some semantic information, the treatment is much easier and faster. Once all tokens have been "cleaned", we proceed to delete the meaningless ones, like articles, prepositions, determinants and so on. To achieve this goal, we have opted for using a stopwords list that contains words that are common in English and then do not provide distinctive meaning by themselves. In particular, our list contains 335 stopwords 
(see Appendix A). At the end, the noise, and then the initial vocabulary, are reduced, which speeds up the processing of the collection and increases the accuracy of the system.

Before proceeding to the second preprocessing step, we have to represent every document, and its filtered tokens, in a computational way. We have decided to use $n$-dimensional vectors, being $n$ the size of the whole collection vocabulary. Every dimension, also called feature, corresponds to a token, and its content is the frequency of this token in the document (see Fig. 1).

By this, the vector can be seen as a quantitative digest of the document it represents. This way of representing documents is known as Vector Space Model (VSM) [22], which is fundamental to many operations in Information Retrieval such as document clustering. In fact, VSM allows to easily figure out the similitude between two documents by computing the dot product (also know as inner product) of their normalized unit vector representations $\vec{v}\left(d_{1}\right)$ and $\vec{v}\left(d_{1}\right)$. The vectors representing the two documents, $\vec{V}\left(d_{1}\right)$ and $\vec{V}\left(d_{2}\right)$, are normalized to unit vectors as this measure of similitude between two documents, named cosine similarity, is equivalent to the cosine of the angle $\theta$ formed by the two vectors, and thus the result must be between 0 and 1 (negative are not possible as the features of the vectors only can contain positive or zero frequencies). Figure 2 and Eq. (1) illustrate how similarity is calculated through

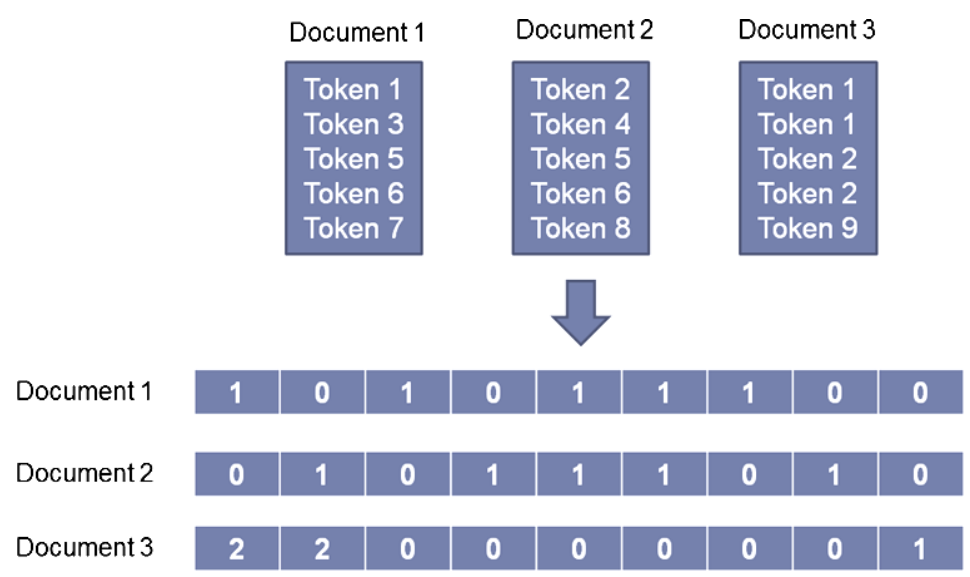

Fig. 1. Example of vectorization of 3 documents with the Vector Space Model algorithm. (Colors are visible in the online version of the article; http://dx.doi.org/10.3233/ISU-130698.)

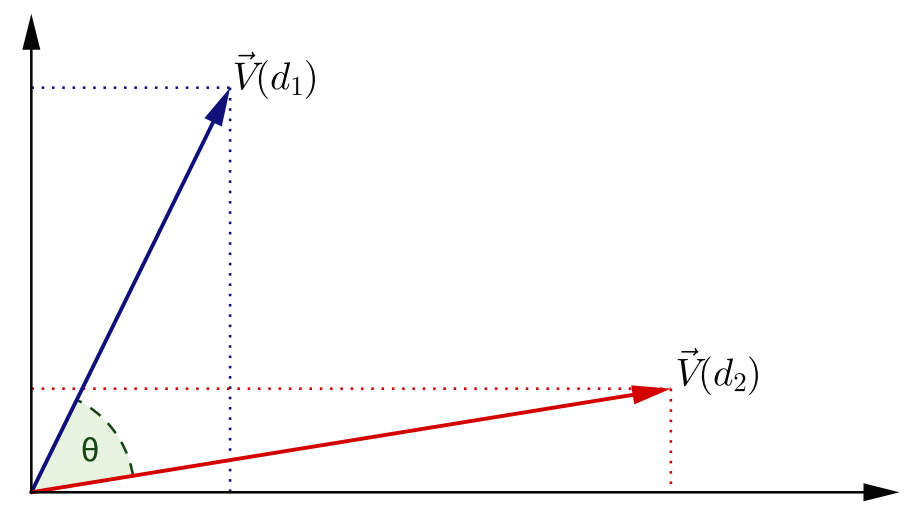

Fig. 2. Cosine similarity metric. (Colors are visible in the online version of the article; http://dx.doi.org/10.3233/ISU-130698.) 
the cosine metric:

$$
\operatorname{sim}\left(d_{1}, d_{2}\right)=\vec{v}\left(d_{1}\right) \cdot \vec{v}\left(d_{2}\right)=\frac{\vec{V}\left(d_{1}\right) \cdot \vec{V}\left(d_{2}\right)}{\left|\vec{V}\left(d_{1}\right)\right|\left|\vec{V}\left(d_{2}\right)\right|}=\frac{\sum_{i=1}^{n} \vec{V}_{i}\left(d_{1}\right) \times \vec{V}_{i}\left(d_{2}\right)}{\sqrt{\sum_{i=1}^{n} \vec{V}_{i}\left(d_{1}\right)^{2}} \times \sqrt{\sum_{i=1}^{n} \vec{V}_{i}\left(d_{2}\right)^{2}}}
$$

Having this measure of similitude for every pairwise documents in the collection, the measure of distance can be obtained directly by calculating distance $=1-$ similarity. This way, the more similar are two documents, the more their distance will be close to 0 and, on the other hand, the more they are different, the more their distance will be close to 1 . This measure of similitude (and hence the distance) is statistical in nature as it only reflects the proportion of tokens that both documents have in common, independently of their semantic meaning. Therefore, it will not reflect faithfully if they share the same topic, but only the rate of words they share. Even if this may seem a drawback, our objective is not to obtain very precise similitude values, but to obtain good enough ones in the shortest possible time.

After this, the system assigns each document to a thematic category or group. For this, we use an unsupervised machine learning method to group documents based on their similarity measures. In this work the $k$-means algorithm [13] is chosen, as it is probably the simplest clustering algorithm available and it matches our needs: it is possible to dynamically select the desired number of clusters (configuring this option is left up to the user, so that the granularity in thematic classification will depend on his needs) and it is sufficiently fast for small sized datasets (so that it can be repeatedly executed at the user's will), allowing the reconfiguration of the clusters in real-time. On the contrary, $k$-means is not a deterministic algorithm because of the random selection of the initial seeds, and then the algorithm does not always converge to the same clusters [5]. This is not acceptable for an interactive system as the user could get confused. That's why we have slightly modified the classical $k$-means algorithm, by executing it $n$ times, each of them with a different random combination of initial seeds, and choosing the ones that, after executing $k$-means, minimize the sum of the distances between any document and the centroid of the group to which it belongs, also called Residual Sum of Squares (RSS).

Finally, in this step we also extract a representative keyword for each document, by retrieving from every vector the feature with the highest value, which represents the meaningful token most frequent in the document. Again, this process is not very precise, but instead it is really simple and fast, which is our goal.

\subsection{Assignment of visual characteristics}

For this work, three attributes of interest were considered: the similarity between documents, the thematic category of each document and the keywords extracted. Taking into account the considerations mentioned in [24], three different visual characteristics were chosen in order to visually represent these attributes.

First, the whole document collection is represented by a three-dimensional cloud of spheres, each of them representing a single document. Similarity between documents is visually represented by the spatial distance between the spheres. So, the more the documents are thematically similar, the closer are their associated spheres. The thematic category is represented by the hue component of color, so that the same category documents have the same color. The hue values of each document are updated every time the number of clusters changes. For the visualization of the keywords, a single textual label is imposed on the sphere. 
Mapping the spatial position of each document requires to calculate its three coordinates in the space. These coordinates can be obtained by reducing the $\mathrm{n}$-dimensional vectors to three-dimensional vectors, or by exploiting other metadata given by the preprocessing phase. In any case, even if vectors' dimensionality is reduced, the relationship between the documents must be maintained. In our case, we opt for using the similarity measures between documents to figure out the 3D coordinates of the spheres. For this purpose, we use the Force-Directed Placement (FDP) technique, that was originally proposed in [7] and later improved in [10]. This spatial projection technique consists in the simulation of attraction and repulsion forces (calculated according to the similarity measure between every pair of documents) among the documents in the collection. The action of these forces results in a spatial layout of documents where the original similarity relationships are visually represented. Although the FDP approach has big scalability issues for big collections, it also has many desirable properties for our goals: good quality of the resulting layout, iterative and real-time positioning process that allows perceiving the documents' movement across space from their initial positions to the final ones (visual feedback that helps user understanding the consequences of his manipulation on the collection), and the ability to extend the force concept to include other factors in the positioning process, as it will be explained in Section 3.2.

Even if our system is three-dimensional, the visualization is projected in two dimensions in the projection screen. Because of this, depth is difficult to be perceived, and then user can be confused about how close his fingerprints are from a specific sphere. To overcome this problem, we provide an easy and intuitive visual feedback that helps user understanding how deep are located his fingerprints. On the one hand, we draw transparent lines from the fingerprints to all the spheres that are located under a predefined threshold. On the other hand, when a sphere is close enough to be selected, a thicker, dotted and colored line (the same color as the sphere) appears to indicate the user that it is selectable.

\section{Interacting with 3D document collections}

Besides the visualization of the documents in the 3D environment, it is essential to provide the user with some techniques to navigate and explore the environment. The classical interaction approach is guided by Shneiderman's famous mantra: Overview first, Zoom, Filter and Details-on-Demand [25]. This principle seems to describe the natural way in which any user explores the information, independently of the environment. In the first step, user should access the overview, providing the general structure behind the information, just like a map does. In the second step, once he has identified the interest areas from the overview, he should be able to focus on these areas, while the other areas come out of the focus in order to reduce the cognitive load and do an efficient use of the screen space. For this goal, user should be able to apply some filters allowing him to keep in the visualization only those documents that are likely to interest him. Finally, user should be able to request and obtain more information related or contained into these filtered documents. Accessing details should be done incrementally on user's demand.

General interaction mechanisms in Virtual Environments can be categorized into 4 main tasks: navigation, selection and manipulation, system control and symbolic input. Navigation techniques allow users to fully explore the environment and to reach any point within it. This allows exploring the information across the different levels of abstraction. At the overview level, it aids users to identify the potential interest areas of information, as done by the FishEye technique [11]. Selection and Manipulation techniques allow users to effectively interact with the elements of the Virtual Environment. Once the user has selected one or more elements of the Virtual Environment, he can manipulate them by modifying their 
position and/or their orientation. System control techniques deal with the modification of the state of either the system or its elements. An example is the removal of a set of elements from the environment or the saving of the current state of the system. Finally, symbolic input techniques allow the user inputting alphanumeric values to the application. These mechanisms must be designed taking into account the user's needs together with the tasks he has to carry out and the specific context of application. Here we present two innovative interaction techniques allowing to manipulate the visual mapping, by improving the identification of thematic groups and the visual perception of document similarities.

\subsection{Modifying the number of document groups}

The first proposed manipulation mechanism allows users to interactively modify the number of potential thematic groups (see Fig. 3(a)).

This means that the last step of the documents' preprocessing must be redone dynamically every time the user changes the number of groups $k$. The interest of this technique is based on the assumption that every user has different cognitive and perceptual capabilities, and different preferences. Some users might want to split the document collection into as many specific groups as possible, while others might prefer less and more general groups. The need for higher or lower subdivision even depends on the user task (e.g. building a mental model of the collection vs. searching for a specific document) or on the phase in the pursue of a certain goal (e.g. filter irrelevant information vs. select the best possible source

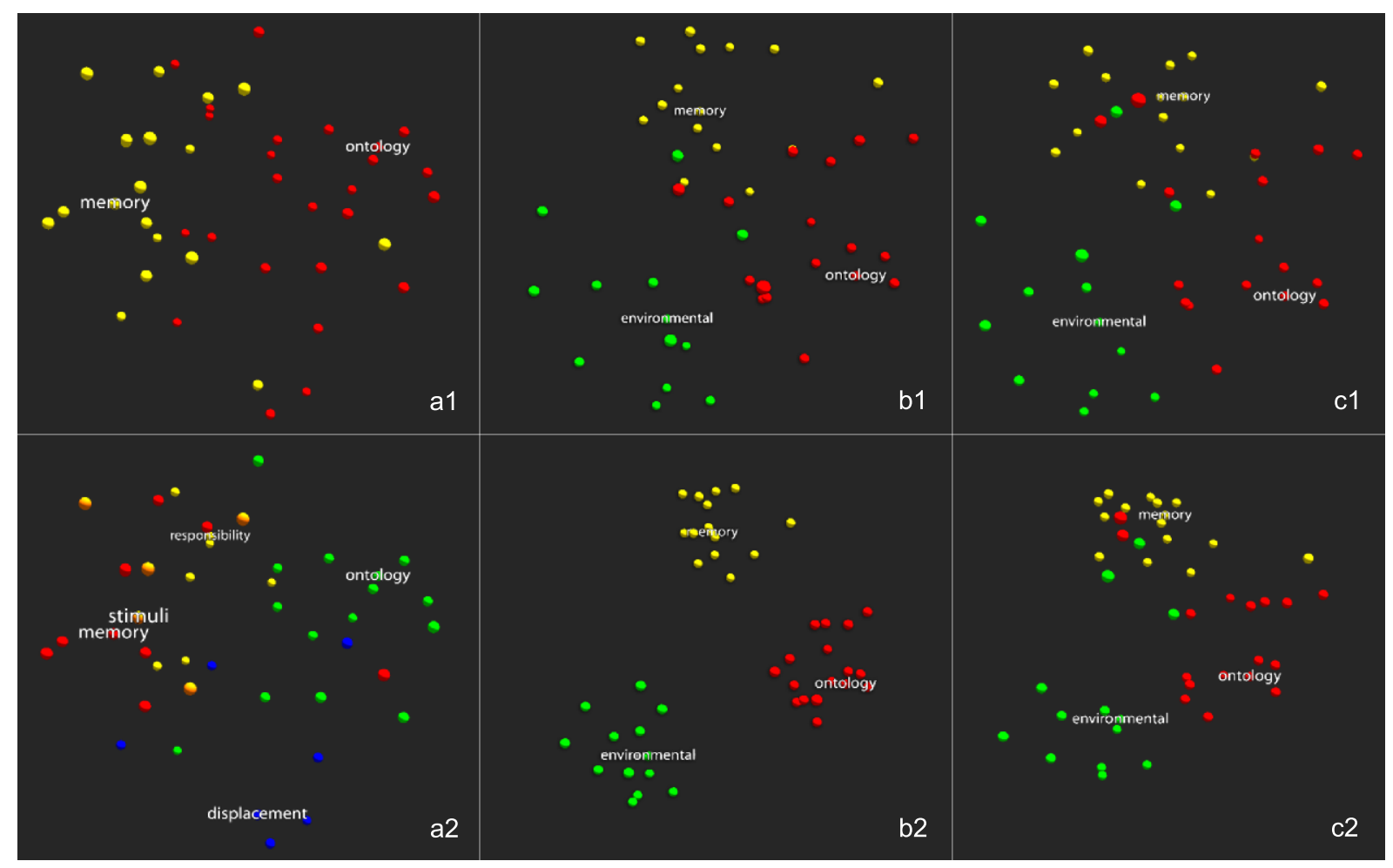

Fig. 3. (a) Changing from two groups (a1) to five groups (a2); (b) Increasing the intra-cluster force from zero (b1) to maximum value (b2); (c) Increasing the inter-documents force from zero (c1) to maximum value (c2). (Colors are visible in the online version of the article; http://dx.doi.org/10.3233/ISU-130698.) 
to search a specific information). Our hypothesis is that combining this mechanism with some document filters can greatly increase the flexibility of the system while exploring or searching.

From the technical perspective, this mechanism can be triggered by the user explicitly and implicitly. In the explicit case, user is able to control the number of groups by manipulating some interface controls. In the implicit case, the number of groups can change when user applies other mechanisms like document or group filtering. In both cases, the clustering algorithm (see Section 2.1) needs to be re-executed for the number of groups expressed through variable $k$.

\subsection{Adjustment of attraction forces}

The second manipulation mechanism we propose allows the user to modify the mapping of the documents' spatial positioning, which is the most important visual characteristic. Although the spatial positioning reflects by itself the similarity between documents, we think that visual clarity of the representation can be improved. In this sense, we propose a modification to the original FDP algorithm described in Section 2.2, called FDP with Force Control (FDPFC) algorithm, where two new attraction forces, dynamically modifiable by the user through interface controls, are added.

The first force modifier aims at enhancing the overview visualization. To achieve this, it modifies the spatial position of spheres so documents that belong to the same cluster get closer and are separated from the other clusters (see Fig. 3(b)). This modification results into a more compact and lean clusters' visualization from the overview perspective. Technically, this effect is generated by adding a specific multiplier to the force that controls the attraction between any pairwise documents, and that applied if and only if both documents belong to the same cluster.

The second force modifier aims at enhancing the inter-document similarity perception, both in the overview and the detail levels. This is accomplished by modifying the spatial position of the spheres so documents whose similarity is higher than a predefined threshold (and then are very similar) are located closer in space, independently of their previous clustering (see Fig. 3(c)). The result is that user can determine at a glance which documents are similar even if they belong to different clusters, and this allows him to determine if the number of clusters allows representing faithfully the collection's distribution, or if the clustering could be improved. For example, if most of the spheres from two clusters are very close, probably their topic is mostly the same, and then they would conflate if the number of groups was decreased. On the contrary, if some spheres of more than one group are very close, probably they would form a new cluster if the number of groups in $k$-means was increased. Also in this case, the effect is implemented by adding a multiplier to the attraction force between pairwise documents if and only if the distance between them is lower than the mean distance between any other pairwise documents.

\subsection{A gesture-based interaction paradigm}

As for our daily tasks we use our hands, we think that the manipulation of $3 \mathrm{D}$ environments is also more intuitive using them. Therefore, we propose a gestural interaction paradigm where the index and the thumb, equipped with reflective solitary markers, are optically tracked to obtain their spatial position and their orientation. In order to handle different interaction techniques only with two fingers, three gestures have been considered. These gestures are identified according to the distance between both fingers: maximal distance (thumb and index spread, see Fig. 4(a)), medium distance (relaxed fingers, see Fig. 4(b)) and minimal distance (thumb and index pinched, see Fig. 4(c)). As distance between fingers 

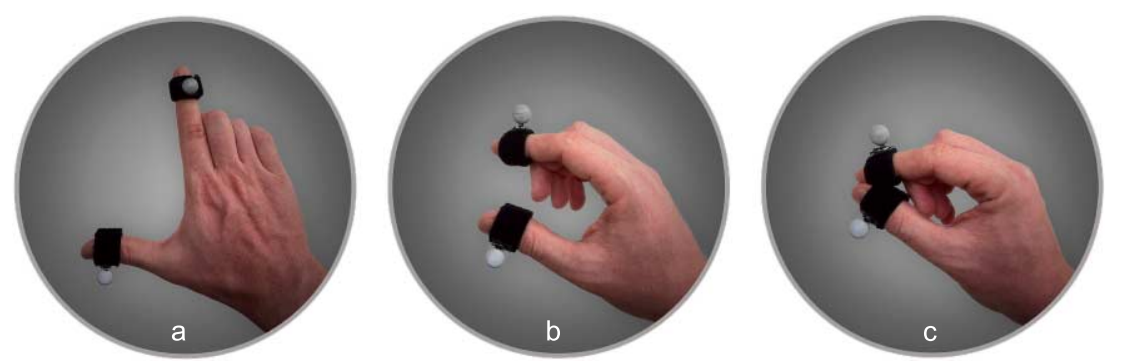

Fig. 4. (a) Maximal distance gesture; (b) Medium distance gesture; (c) Minimal distance gesture. (Colors are visible in the online version of the article; http://dx.doi.org/10.3233/ISU-130698.)

depends absolutely on users' physical characteristics, an initial training phase is carried out to obtain the individual finger distances (minimal, medium and maximal) of every user. This is simply done by following screen instructions and mimic shown finger poses for minimal, normal and maximal finger gestures. Fingers are virtually represented by two 3D fingerprints (see Fig. 5(a)), that are immediately identified by the user as representations of his fingers because of the realtime feedback received in terms of position, inclination and rotation while the real fingers move. Moreover, a textual label is drawn between the fingers to indicate the user which is the current gesture.

In order to test our approach, we have implemented a prototype system providing mostly all of the interaction techniques explained in Section 3. First, we offer full navigation techniques, that handle 6 Degrees Of Freedom: 3 DOF corresponding to the translation of the viewpoint along the axis $x, y$ and $z$ (horizontal and vertical translations, and zoom in and out), and 3 DOF mapped to the rotation of the viewpoint with respect to these three axis. In the first case, user can translate the viewpoint using the maximal gesture (see Fig. 4(a)), while rotation is carried out when user pinches his fingers (see Fig. 4(c)). The medium distance gesture is used for free movements of user's hands without affecting in any way the viewpoint or any other element in the Virtual Environment. Then, user is allowed to reach any point of the environment and to visualize the collection with the desired detail. Associated to the zooming mechanisms, and aiming at avoiding to cognitive overload the visualization, keywords get visible only when the distance between the spheres they are attached to and the position of the user's avatar (fingerprints) is under a defined threshold. The second group of interaction techniques deals with the selection of one or more documents, as our system is intended both for individual document search and global exploration of a collection of documents. The first one permits selecting one single document, whereas the latter allows selecting all the documents that belong to the same cluster. For increased flexibility, the system allows making incremental selections, that is adding new documents and/or groups to the current selection. We also provide the possibility of deselecting a group that has been previously selected. In all these cases, when a sphere is selected, it gets highlighted to provide a visual feedback to the user. With respect to the manipulation, no techniques have been implemented as the user does not have to manually translate spheres (this is automatically done by the FDP algorithm, and by modifications added in the FDPFC), and spheres' rotation is pointless because of their inner shape. At last, some system control techniques are proposed to provide the user with eight different functionalities for manipulating the collection, implemented as pop-up widgets (see Fig. 5). To avoid undesired executions of these functionalities during navigation, widgets have to remain activated by the user for at least 2 seconds in order to become operational. Most of the widgets have a binary behaviour and their associated functionality is executed just by activation. The first case is jumping between groups in order to focus the visualization over the centroid of one group (see Fig. 5(i)). If more than one group 


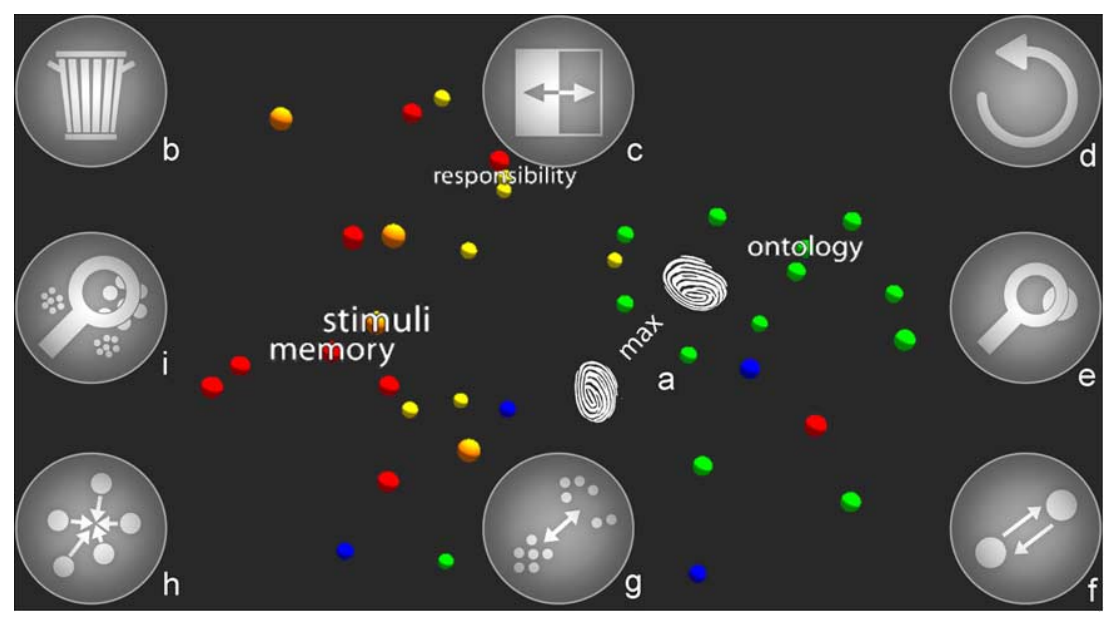

Fig. 5. User interface: (a) Virtual fingerprints; (b) Delete; (c) Invert; (d) Undo; (e) Inspect; (f) Document force adjustment; (g) Groups adjustment; (h) Group force adjustment; (i) Jump. (Colors are visible in the online version of the article; http://dx. doi.org/10.3233/ISU-130698.)

has been created, every activation results in focusing the viewpoint on a new group, which is a great help when navigating and exploring over groups and not over individual documents. It also acts like an anchor when the user gets lost in the environment and is not able to locate the spheres. Secondly, we make possible to consult in a new window the content of a document that has been selected previously (see Fig. 5(e)). Thirdly, the user can invert a selection (see Fig. 5(c)), which is very useful when the user wants to select all the groups or individual spheres apart from one. Furthermore, when one or more documents or groups are selected, the system offers the possibility of clearing them from the visualization (see Fig. 5(b)). This means that they are not considered in clustering, projection and visualization algorithms but they are not physically deleted. Additionally, the last document(s) cleared can be restored in order to consider them again in clustering, projection and visualization algorithms (see Fig. 5(d)). The last three functionalities require inputting a numerical value, and then a symbolic input technique is also required. So, besides activation, their widgets require the user to indicate a numerical value, which is done through finger movements: every medium-to-maximal gesture movement implies increasing by one unit the corresponding value, while pinching the fingers (medium-to-minimal gesture movement) translates to decreasing the corresponding value by one unit. The first functionality that requires inputting a numerical value is the dynamic adjustment of the number of groups for $k$-means (see Section 3.1), while the two others correspond to the adjustment of both force modifiers added in the proposed FDPFC approach (see Section 3.2).

\section{Experimental evaluation}

\subsection{Experiment design}

A user evaluation was designed in order to evaluate the impact of the modifications introduced by the FDPFC. An experiment was conducted in which one independent variable, the type of 3D mapping method, was manipulated with two possible levels: Force Directed Placement (FDP), and Force Directed Placement with Force Control (FDPFC). A total of 36 subjects were selected for the experiment and randomly assigned to one of the two experimental treatments. All subjects were computer science students 


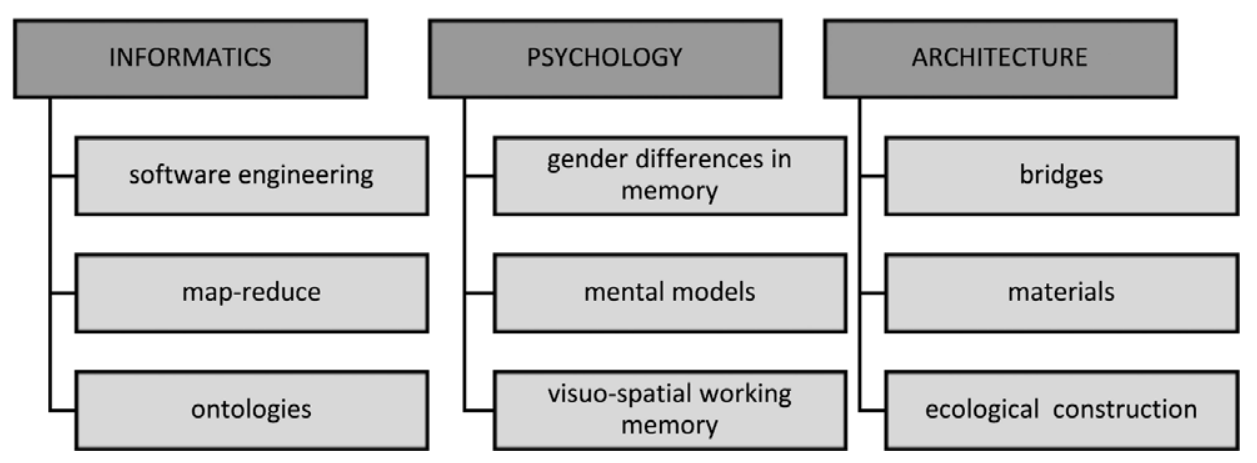

Fig. 6. Structure of the experimental document collection.

or professors, so they were assumed to be able to easily understand the abstraction applied in the 3D representation of document collections, and to quickly learn and apply new interaction techniques. Ages of the participants ranged from 21 to 40, with 25 males and 9 females. The measured dependent variables were: Precision in the mental model resulting from exploration $(\mathrm{P})$, Effectiveness in search (E), and Satisfaction (S). The null hypotheses were:

H01. The mean precision in the mental model obtained from exploration is the same for FDP and FDPFC.

H02. The mean effectiveness in search is the same for FDP and FDPFC.

H03. The mean satisfaction is the same for FDP and FDPFC.

Statistical $t$-tests were applied to evaluate these hypotheses.

Precision of the mental model was evaluated after an exploration task. The experimental document collection was manually composed by intentionally selecting documents within a set of nine predefined topics which in turn belonged to three main areas. The designed taxonomy for the collection is reflected in Fig. 6. Precision of the mental model was measured by presenting, after some minutes of free exploration, a brief thematic questionnaire (see Appendix B) with nine questions in the form "Were there any documents in the collection talking about topic?" A five levels response scale was presented with the meaning -2: "definitely no", -1 : "probably no", 0 : "I do not know", 1: "probably yes" and 2: "definitely yes". The reference topics for the nine questions were selected so that some of them were present in the collection, some of them were not present in the collection, and some of them were not present but related to topics present in the collection:

Present: Ontologies, Bridges and Memory.

Not present: Animals, Art and Civilizations.

Not present, but related: Buildings, Pedagogy and Software agents.

The hypothesis was that the availability of force control mechanisms in FDPFC would allow the subjects to construct a more precise model of the thematic structure of the collection, particularly by strengthening the intra-cluster attraction force.

Effectiveness in search was measured via a search task in which the participant was asked to find within the collection a representative document talking about a particular topic, as well as two additional documents that were the closest to it in their thematic content. The best possible solution for the task was pre-calculated. Taking the inter-document distance matrix filled by applying the cosine similarity measure to every pair of documents, the effectiveness in search was computed as the distance between 
the solution document provided by the experimental subject and the pre-calculated optimal solution. Instead of requesting just one representative document, two additional neighbors were also demanded (see Appendix C). For the evaluation of the selected neighbors we decided to count how many of them matched with the optimal neighbors, also pre-calculated for a given topic. The hypothesis was that the availability of force control mechanisms in FDPFC would allow the subjects to find a higher quality set of documents, particularly by strengthening the inter-documents attraction force. We hypothesized that the increase in quality would be more significant when considering the neighbors than just by comparing the best representative.

Finally, User Satisfaction was evaluated by a questionnaire at the end of the experience. Fifteen questions were common for both experimental groups, with three additional questions for the FDPFC group evaluating the perceived usefulness of the force control capabilities (see Appendix D), and two additional questions for the FDP group trying to find out the usefulness they anticipated for these features (see Appendix D). There were four open questions investigating previous user knowledge of document collection exploration and search tools, and requesting a general evaluation of positive and negative aspects. The remainder questions offered a five levels response scale going from 0 to 4 , in which 0 was interpreted as "I strongly disagree", 1 represented "I disagree", 2 meant "Neutral", 3 stood for "I agree", and 4 meant "I strongly agree". Also, the full interaction of each participant with the system was video recorded for further analysis, mainly focusing on a qualitative evaluation of the proposed interaction techniques.

The experiment was conducted along four days, with half an hour assigned for each subject. Each subject went through the following stages:

(1) Preparation. Before entering the experimental area a document was handed over in which a general description of the experiment's goal and procedure was presented, to ensure that all the participants understood their role and were properly prepared.

(2) Training. The participant was led to the projection screen and ten minutes were allocated for the participant to familiarize with the visualization display and interaction techniques. A training collection was presented and the participant was explained how to interpret the display and how to interact with the collection.

(3) Exploration. The test collection was loaded and the participant was instructed to freely explore the collection trying to get an idea of the documents' topics. Five minutes were left for this stage.

(4) Mental model evaluation. The participant was taken to a separate room and five minutes were left for completing the brief thematic questionnaire.

(5) Search evaluation. The participant was taken again in front of the projection screen, and five minutes were left for the search task.

(6) User satisfaction evaluation. Again at a separate room, the participant was requested to complete the satisfaction questionnaire and was thanked by his collaboration. No time limit was imposed at this stage.

\subsection{Experimental setting}

The test arrangement consisted of a $2 \times 1.5$ meter screen, bottom side on a level of one meter (see Fig. 7(a)). The subjects were standing in one and a half meter in front of it. For tracking fingertips we are using an optical marker based approach. We have an optical infra-red based tracking system from Naturalpoint installed around the screen. Four cameras, each in a corner of the screen, directed to the subjects home position (see Fig. 7(b)). Each camera is a V100:R1 model, allowing capturing at $100 \mathrm{fps}$ 


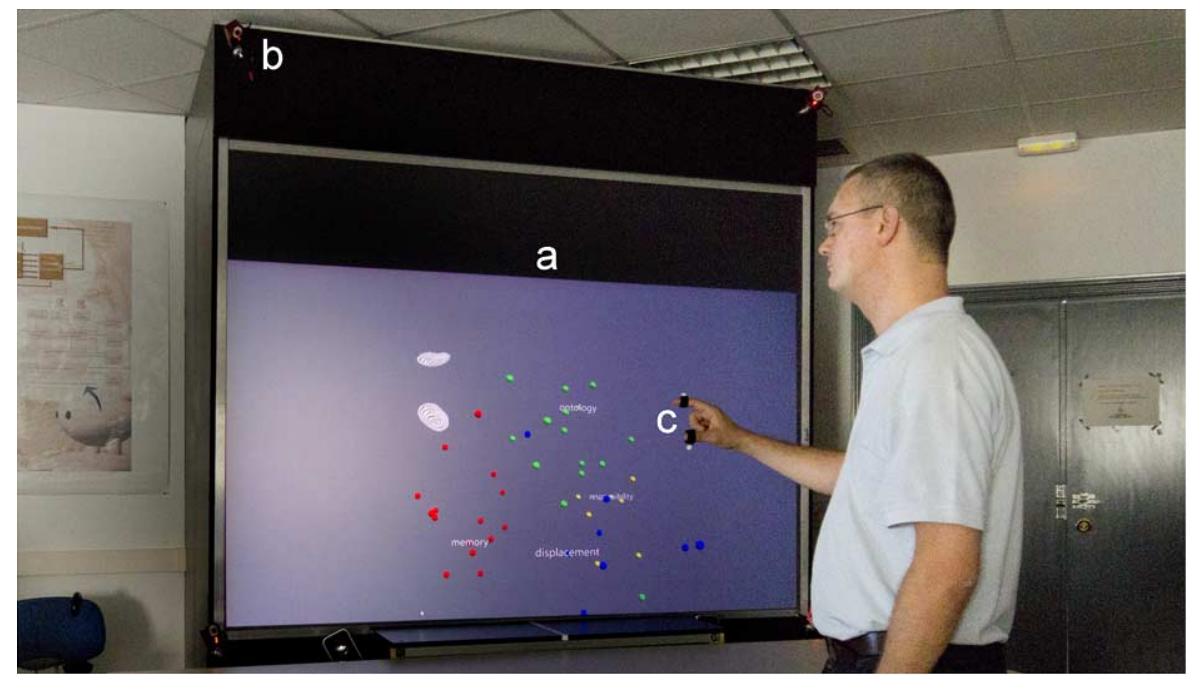

Fig. 7. VR installation: (a) Stereoscopic screen; (b) Optical trackers; (c) Optical markers. (Colors are visible in the online version of the article; http://dx.doi.org/10.3233/ISU-130698.)

with $640 \times 480$ resolution and subpixel precision. The smaller the chosen working area is, the better is the $3 \mathrm{D}$ resolution of tracked objects. Therefore we adjusted the camera system so that the subject had an active working range of $1.5 \times 1.5$ meters.

The default software from Naturalpoint's tracking system is geared to handle primarily rigid body objects. These objects have multiple reflective markers attached, at least three. The constellation of these markers is trained into the tracking system and can hence be recognized. For our arrangement we decided not to use those marker constellations for tracking objects. They have a considerable size, not smaller than $5 \times 5$ centimeters, therefore we chose to use solitary markers to track index and thumb fingers (see Fig. 7(c)). Each solitary marker has a diameter of only $1.1 \mathrm{~cm}$ and then can be easily attached to a fingertip. Naturalpoint's own UDP streaming protocol permits also the access to solitary marker information. By receiving all of the untracked markers we have full control over them, but we also gained the task of filtering and taking care of the tracking process by ourselves. Occasionally false solitary markers are detected by the tracking system. They are caused by inadvertent reflections in the scene like from shiny metal surfaces or eyeglass lenses. Those false positives usually appear only for a snatch. To distinguish the two solitary markers of the two fingers of our proband we must keep track of all solitary markers in the scene and shortlist those who have a sufficing long track history.

\subsection{Results and discussion}

\subsubsection{Precision of the mental model}

Table 1 shows the mean response value for each of the Likert format questions in the thematic questionnaire (column (A) for FDP and (B) for FDPFC), as well as the total number of correct responses (column (C) for FDP and (D) for FDPFC). If a topic is certainly included in the collection (see column (E)), the correct response is considered to be 2, while it should be -2 if the topic is not in the collection. Any other response is considered incorrect (that is why the numbers of correct answers are very low in some cases). The mean value helps to visualize how far from being correct were the responses in average. 
Table 1

Thematic questionnaire

\begin{tabular}{lccccc}
\hline Topic & (A) FDP mean & (B) FDPFC mean & (C) FDP correct & (D) FDPFC correct & (E) In collection \\
\hline Ontologies & 1.9 & 1.8 & 17 & 15 & YES \\
Bridges & 1.1 & 1.4 & 11 & 12 & YES \\
Memory & 1.4 & 1.7 & 10 & 12 & YES \\
Animals & -0.6 & -0.6 & 4 & 4 & NO \\
Art & -0.7 & -0.7 & 2 & 4 & NO \\
Civilizations & -0.3 & -0.1 & 1 & 4 & NO \\
Buildings & 0.3 & 0.8 & 0 & 1 & RELATED \\
Pedagogy & 0.3 & -0.1 & 0 & 2 & RELATED \\
Software agents & 0.8 & 0.8 & 0 & 0 & RELATED \\
TOTAL & & & 45 & 54 & \\
\hline
\end{tabular}

The topics that were certainly in the collection (Ontologies, Bridges and Memory) have been noticed by the majority of the users, with more than 10 out of 18 subjects in each group (with classical FDP and with FDPFC) giving the correct answer (2). If we consider topics that were not included in the collection and were semantically quite far from other topics in the collection (Animals, Art and Civilizations), the percentage of correct responses is low (4 out of 18 at most, again in both groups) but we can notice less uncertainty in the group of users who could control the attraction forces (FDPFC), with higher success rates. In the case of topics that were not in the collection but were intentionally close to other topics positively included in the collection (Buildings, Pedagogy and Software agents), the success rate is the lowest, as expected, with a slightly highest success rate in the FDPFC group. The test on the equality between the mean number of correct responses in the FDP and FDPFC conditions did not allow us to reject the null hypothesis $(\sigma=0.32)$.

\subsubsection{Effectiveness in search}

Two search tasks were demanded. The mean distance between the users' solutions and the optimal one was similar in both groups $(0.29$ for FDP vs 0.26 for FDPFC in the first task, and 0.09 for FDP vs 0.11 for FDPFC in the second one). For the evaluation of the selected neighbors we decided to count the number of "good neighbors" provided, considering a good neighbor one of the two closest documents to the one chosen as the best (for the specific solution posed by each user). Being 36 the maximum possible group score at each search task (18 participants by 2 good neighbors), the obtained results ranged from 17 to 19 in each of the four cases ( 2 search tasks by 2 experimental groups). No significant difference was found between FDP and FDPFC.

\subsubsection{Satisfaction}

If we analyze the mean response value for each of the Likert format questions in the satisfaction questionnaire, we can see that all means are higher than 2 (the neutral point in the scale). For the overall questionnaire, the mean response value for the FDP group is 3.12, while it is 3.29 for the FDPFC group, slightly better but still the difference is not significant $(\sigma=0.13)$. The opinion of the users towards the system is quite positive, with the most remarkable positive opinion (above 3.5 points) reflected in questions Q9 (interrogating about the usefulness of keyword visualization), Q15 (dealing with the possibility to jump the viewpoint from one group to another) and Q18 (demonstrating the belief in the possibility to make more benefit from the system with more training and experience) for both experimental conditions, and also in question Q13 (about the possibility to select a group of documents and remove it) for the 
Table 2

Satisfaction questionnaire - group specific questions

\begin{tabular}{lcc}
\hline Question & FDP mean & FDPFC mean \\
\hline Q16a & 2.56 & \\
Q17a & 2.06 & \\
Q16b & & 3.65 \\
Q17b & & 3.31 \\
Q17b2 & & 3.00 \\
\hline
\end{tabular}

FDPFC group. The most valued feature for both groups was keyword labeling. Table 2 details the mean response values for the FDP specific and FDPFC specific questions.

Regarding the features that were available for the FDPFC group but not for the FDP group it is interesting to see that the latter does not value much the possibility to include them, despite not having any experience with them (with mean values closer to 2-neutral), while the group that effectively had the opportunity to enjoy the features certainly valued much more their usefulness. The difference among the mean values for question pairs Q16a-Q16b and Q17a-Q17b is significant ( $\sigma=0.0003$ in the first pair and $\sigma=0.0023$ in the second pair).

\section{Conclusions and future work}

We presented some contributions towards a more intuitive and effective interaction with 3D visualizations of unstructured document collections. A prototype system has been developed fully automating the information visualization pipeline. A modification of the classical FDP algorithm has been proposed (the FDPFC variant) to determine the spatial position of each document's spherical representation in the 3D space. This modification allows the user to control the attraction forces to be applied among documents so that group separation or inter-document similarity can be visually enhanced, as required for the task at hand. A full set of interaction techniques have been implemented through a new gesture-based interaction paradigm based on tracking the position of two hand fingers (index and thumb) by using solitary markers. Navigation, selection and manipulation of both individual documents and document clusters, as well as adjustment of the visualization settings (number of clusters and attraction forces), can be achieved just with very basic hand movements and a set of reactive zones in space.

After experimentation, FDPFC seemed to help decrease the uncertainty about the thematic structure of the collection, although no conclusive evidence was found that FDPFC allows the construction of a more precise mental model. Partly this could be explained by the fact that the exploration task was the first experience of the users with the system after a really very brief training period (just ten minutes), and the participants may have felt overwhelmed by the new way to interact with the system, the number of functionalities offered and the tasks required. In fact we observed very limited use of the force control options during exploration. We believe that a longer training period in which users have the opportunity to really understand the effect and possibilities of each interaction mechanism could result into more effective use of the force control features and a more significant effect on the precision of the mental model.

Regarding the user satisfaction and subjective perception, keywords were the most valued option. This is probably due to the fact that it was the most evident way to look for the required documents in the search task. Some users applied an almost exhaustive search strategy, inspecting every document's 
keyword, or even explicitly consulting all documents' contents. This strategy could suffice in our limitedsize experimental collection, but we believe that if faced with much more populated collections, users would start to realize the usefulness and higher efficiency of alternative manipulation techniques.

In our future work we plan to experiment with bigger and more complex document collections and with a longer and more explicit training period that really helps users to become proficient in the use of the interaction techniques, to face users to situations in which the application of the new interaction techniques really becomes a worthy alternative to more evident strategies, and to avoid results to be biased by the initial user's disorientation. We also plan to improve the preprocessing phase, in order to obtain better and more sophisticated metadata from documents allowing to classify them better and to obtain better keywords. Finally, we also want to carry on a new experiment using a classical desktop system approach, well known by users, so that they will be able to concentrate only in using the proposed functionalities, and not on learning the new interaction techniques themselves. This will allow us to get sure that results are not affected by the gestural paradigm.

\section{Appendix A. Stopwords list}

\begin{tabular}{|c|c|c|c|c|c|c|c|c|}
\hline $\bar{a}$ & anything & by & elsewhere & further & if & mine & often & seem \\
\hline able & anyway & call & empty & get & in & more & on & seemed \\
\hline about & anywhere & can & enough & give & inc & moreover & once & seeming \\
\hline above & are & cannot & etc & go & indeed & most & one & seems \\
\hline across & around & cant & even & got & interest & mostly & only & serious \\
\hline after & as & co & ever & had & into & move & onto & several \\
\hline afterwards & at & computer & every & has & is & much & or & she \\
\hline again & back & con & everyone & hasnt & it & must & other & should \\
\hline against & be & could & everything & have & its & my & others & show \\
\hline all & became & couldnt & everywhere & havent & itself & myself & otherwise & side \\
\hline almost & because & cry & except & he & just & name & our & since \\
\hline alone & become & de & few & hence & keep & namely & ours & sincere \\
\hline along & becomes & dear & fifteen & her & last & neither & ourselves & six \\
\hline already & becoming & describe & fifty & here & latter & never & out & sixty \\
\hline also & been & detail & fill & hereafter & latterly & nevertheless & over & so \\
\hline although & before & did & find & hereby & least & next & own & some \\
\hline always & beforehand & do & fire & herein & less & nine & part & somehow \\
\hline am & behind & does & first & hereupon & let & no & per & someone \\
\hline among & being & done & five & hers & like & nobody & perhaps & something \\
\hline amongst & below & down & for & herself & likely & none & please & sometime \\
\hline amoungst & beside & due & former & him & ltd & noone & put & sometimes \\
\hline amount & besides & during & formerly & himself & made & nor & rather & somewhere \\
\hline an & between & each & forty & his & many & not & re & still \\
\hline and & beyond & eg & found & how & may & nothing & said & such \\
\hline another & bill & eight & four & however & me & now & same & system \\
\hline any & both & either & from & hundred & meanwhile & nowhere & say & take \\
\hline anyhow & bottom & eleven & front & $\mathrm{i}$ & might & of & says & ten \\
\hline anyone & but & else & full & ie & mill & off & see & than \\
\hline
\end{tabular}




\begin{tabular}{lcccccccc}
\hline that & therefore & though & too & under & we & whereafter & whither & within \\
the & therein & three & top & until & well & whereas & who & without \\
their & thereupon & through & toward & up & were & whereby & whoever & would \\
them & these & throughout & towards & upon & what & wherein & whole & yet \\
themselves & they & thru & twas & us & whatever & whereupon & whom & you \\
then & thick & thus & twelve & very & when & wherever & whose & your \\
thence & thin & tis & twenty & via & whence & whether & why & yours \\
there & third & to & two & wants & whenever & which & will & yourself \\
thereafter & this & together & un & was & where & while & with & yourselves \\
thereby & those & & & & & & &
\end{tabular}

\section{Appendix B. Thematic questionnaire}

\begin{tabular}{|ll|}
\hline Session: & DO NOT FILL THIS BOX \\
\hline
\end{tabular}

\section{Thematic questionnaire}

Indicate to what extent you agree with the following statements:

- Were there any documents in the collection talking about ONTOLOGIES

\begin{tabular}{|l|l|l|l|l|}
\hline$\square$ Definitely NO & $\square$ Probably NO & $\square$ I do not know & $\square$ Probably YES & $\square$ Definitely YES \\
\hline
\end{tabular}

- Were there any documents in the collection talking about BUILDINGS

\begin{tabular}{|l|l|l|l|l|}
\hline$\square$ Definitely NO & $\square$ Probably NO & $\square$ I do not know & $\square$ Probably YES & $\square$ Definitely YES \\
\hline
\end{tabular}

- Were there any documents in the collection talking about BRIDGES

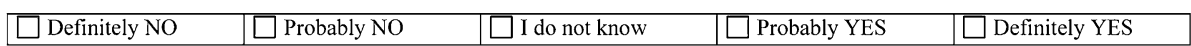

- Were there any documents in the collection talking about ANIMALS

\begin{tabular}{|l|l|l|l|l|}
\hline$\square$ Definitely NO & $\square$ Probably NO & $\square$ I do not know & $\square$ Probably YES & $\square$ Definitely YES \\
\hline
\end{tabular}

- Were there any documents in the collection talking about PEDAGOGY

\begin{tabular}{|l|l|l|l|l|}
\hline$\square$ Definitely NO & $\square$ Probably NO & $\square$ I do not know & $\square$ Probably YES & $\square$ Definitely YES \\
\hline
\end{tabular}

- Were there any documents in the collection talking about SOFTWARE AGENTS

\begin{tabular}{|l|l|l|l|l|}
\hline$\square$ Definitely NO & $\square$ Probably NO & $\square$ I do not know & $\square$ Probably YES & $\square$ Definitely YES \\
\hline
\end{tabular}

- Were there any documents in the collection talking about MEMORY

\begin{tabular}{|l|l|l|l|l|}
\hline$\square$ Definitely NO & $\square$ Probably NO & $\square$ I do not know & $\square$ Probably YES & $\square$ Definitely YES \\
\hline
\end{tabular}

- Were there any documents in the collection talking about ART

\begin{tabular}{|l|l|l|l|l|}
\hline$\square$ Definitely NO & $\square$ Probably NO & $\square$ I do not know & $\square$ Probably YES & $\square$ Definitely YES \\
\hline
\end{tabular}

- Were there any documents in the collection talking about CIVILIZATIONS

\begin{tabular}{|l|l|l|l|l|}
\hline$\square$ Definitely NO & $\square$ Probably NO & $\square$ I do not know & $\square$ Probably YES & $\square$ Definitely YES \\
\hline
\end{tabular}




\section{Appendix C. Search questionnaire}

\begin{tabular}{|lc|}
\hline Session: & DO NOT FILL THIS BOX \\
\hline
\end{tabular}

\section{$\underline{\text { Search Task }}$}

\section{TASK 1:}

Find within the collection the document you consider the most significant for the next topic. Indicate also the two documents you consider most similar to it:

\section{Biological substrate for visuo-spatial working memory}

- Most representative document ID

- Most similar documents IDs

\section{TASK 2:}

Find within the collection the document you consider the most significant for the next topic. Indicate also the two documents you consider most similar to it:

\section{Use of mapreduce to calculate the similarity between two documents}

- Most representative document ID

- Most similar documents IDs 


\section{Appendix D. User satisfaction questionnaire}

\begin{tabular}{|ll|}
\hline Session: & DO NOT FILL THIS BOX \\
\hline
\end{tabular}

\section{Satisfaction Questionnaire}

Once the experimental session has finished, indicate your level of agreement with the following statements:

- This tool is useful to explore collections of documents

\begin{tabular}{|l|l|l|l|}
\hline$\square$ I strongly disagree & $\square$ I disagree & $\square$ Neutral & $\square$ I agree
\end{tabular}$\square$ I strongly agree

- ¿Which alternatives to you know to carry out this exploration task?

- This tool is useful to search a specific document within a collection of documents

\begin{tabular}{|l|l|l|l|}
\hline$\square$ I strongly disagree & $\square$ I disagree & $\square$ Neutral & $\square$ I agree
\end{tabular}

- ¿Which alternatives to you know to carry out this search task?

- It is easy to appreciate that the distance between the documents in the virtual space is directly related to their thematic similarity

\begin{tabular}{|c|c|c|c|c|}
\hline$\square$ I strongly disagree & $\square$ I disagree & $\square$ Neutral & $\square$ I agree & $\square$ I strongly agree \\
\hline
\end{tabular}

- The distance between the documents in the space helps me to better understand the potential thematic groups present in the collection

\begin{tabular}{|c|c|c|c|c|}
\hline$\square$ I strongly disagree & $\square$ I disagree & $\square$ Neutral & $\square$ I agree & $\square$ I strongly agree \\
\hline
\end{tabular}

- The distance between two documents in the space is useful to understand their thematic similarity

\begin{tabular}{|c|c|c|c|c|}
\hline$\square$ I strongly disagree & $\square$ I disagree & $\square$ Neutral & $\square$ I agree & $\square$ I strongly agree \\
\hline
\end{tabular}

- Allowing to select the number of desired groups of documents helps me to better understand the structure of the collection

\begin{tabular}{|l|l|l|l|}
\hline$\square$ I strongly disagree & $\square$ I disagree & $\square$ Neutral & $\square$ I agree \\
\hline
\end{tabular}

- The use of colors helps me to better differentiate the potential thematic groups present in the collection

\begin{tabular}{|l|l|l|l|}
\hline$\square$ I strongly disagree & $\square$ I disagree & $\square$ Neutral & $\square$ I agree \\
\hline
\end{tabular}

- The keywords shown for every document help me to better differentiate the potential thematic groups present in the collection

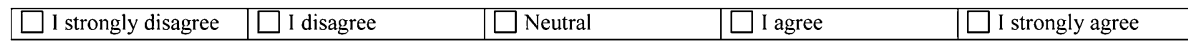

- The keywords shown for every document help me to find a document of interest

\begin{tabular}{|l|l|l|l|}
\hline$\square$ I strongly disagree & $\square$ I disagree & $\square$ Neutral & $\square$ I agree \\
\hline
\end{tabular}


- I found it useful to be able to navigate through the space occupied by the documents (do not answer this question if you did not use this functionality)

\begin{tabular}{|l|l|l|l|l|}
\hline$\square$ I strongly disagree & $\square$ I disagree & $\square$ Neutral & $\square$ I agree & $\square$ I strongly agree \\
\hline
\end{tabular}

- I found it useful to be able to select and clear a group of documents (do not answer this question if you did not use this functionality)

\begin{tabular}{|l|l|l|l}
\hline$\square$ I strongly disagree & $\square$ I disagree & $\square$ Neutral & $\square$ I agree \\
\hline
\end{tabular}

- I found it useful to be able to select one or more documents and invert the selection (do not answer this question if you did not use this functionality)

\begin{tabular}{|c|c|c|c|c|}
\hline$\square$ I strongly disagree & $\square$ I disagree & $\square$ Neutral & $\square$ I agree & $\square$ I strongly agree \\
\hline
\end{tabular}

- I found it useful to be able jump from one group to another (do not answer this question if you did not use this functionality)

\begin{tabular}{|c|c|c|c|c|}
\hline$\square$ I strongly disagree & $\square$ I disagree & $\square$ Neutral & $\square$ I agree & $\square$ I strongly agree \\
\hline
\end{tabular}

\begin{tabular}{|c|c|c|c|c|}
\hline \multicolumn{4}{|c|}{ - 1 would have liked to be able to make more distant the different groups of documents } & \multirow{2}{*}{$\begin{array}{l}\text { Only in FDP group } \\
\square \text { I strongly agree } \\
\end{array}$} \\
\hline$\square$ I strongly disagree & $\square$ I disagree & $\square$ Neutral & $\square$ I agree & \\
\hline \multicolumn{5}{|c|}{ - I would have liked to be able to identify with more clarity the documents similar to each other } \\
\hline$\square$ I strongly disagree & $\square$ I disagree & $\square$ Neutral & $\square$ I agree & $\square$ I strongly agree \\
\hline
\end{tabular}

\begin{tabular}{ll}
\hline & Only in FDPFC group
\end{tabular}

(do not answer this question if you did not use this functionality)

\begin{tabular}{|l|l|l|l|}
\hline$\square$ I strongly disagree & $\square$ I disagree & $\square$ Neutral & $\square$ I agree \\
\hline
\end{tabular}

- I found it useful to be able to adjust the attraction force between similar documents (do not answer this question if you did not use this functionality)

\begin{tabular}{|l|l|l|l}
\hline$\square$ I strongly disagree & $\square$ I disagree & $\square$ Neutral & $\square$ I agree \\
\hline
\end{tabular}

- I consider that visualizing the movement of the documents while adjusting the attraction forces has helped me to better understand what was going on

\begin{tabular}{|l|l|l|l|}
\hline$\square$ I strongly disagree & $\square$ I disagree & $\square$ Neutral & $\square$ I agree \\
\hline
\end{tabular}

- With more experience and training, l could take much more advantage of the application

\begin{tabular}{|l|l|l|l|}
\hline$\square$ I strongly disagree & $\square$ I disagree & $\square$ Neutral & $\square$ I agree
\end{tabular} I strongly agree

- The aspects I most value in the system are:

- The aspects that should be improved in the system are: 


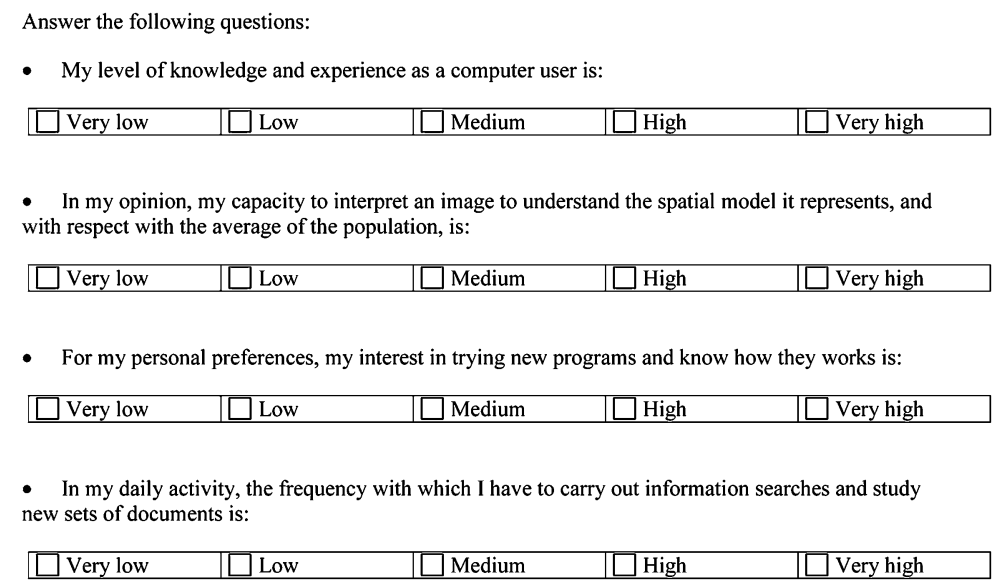

\section{References}

[1] S. Abney, Part-of-Speech Tagging and Partial Parsing, Kluwer, 1996, pp. 118-136.

[2] S. Benford and J. Mariani, Virtual environments for data sharing and visualisation - populated information terrains, in: Populated Information Terrains, Research Report CSCW/5/94, SECAMS, Springer-Verlag, 1994, pp. 168-182.

[3] E. Brill, A simple rule-based part of speech tagger, in: Proceedings of the Workshop on Speech and Natural Language, HLT'91, ACL, Stroudsburg, PA, USA, 1992, pp. 112-116.

[4] S.K. Card, J.D. Mackinlay and B. Shneiderman, Readings in Information Visualization: Using Vision to Think, Morgan Kaufmann Pub., San Francisco, CA, USA, 1999.

[5] I. Davidson and A. Satyanarayana, Speeding up $k$-means clustering by bootstrap averaging, in: Proceedings of the Workshop on Clustering Large Data Sets at ICDM'03, 2003.

[6] J.L. Dawson, Suffix removal for word conflation, Bulletin of the Association for Literary and Linguistic Computing 2(3) (1974), 33-46.

[7] P. Eades, A heuristic for graph drawing, Congressus Numerantium 42 (1984), 149-160.

[8] K. Fairchild, S. Poltrock and G. Furnas, SemNet: Three-Dimensional Graphic Representations of Large Knowledge Bases, Cognitive Science and Its Applications for Human-Computer Interaction, Lawrence Erlbaum Associates, 1988.

[9] R. Florian, A. Ittycheriah, H. Jing and T. Zhang, Named entity recognition through classifier combination, in: Proceedings of the 7th Conference on Natural Language Learning at HLT-NAACL 2003, CONLL'03, Vol. 4, ACL, Stroudsburg, PA, USA, 2003, pp. 168-171.

[10] T.M.J. Fruchterman and E.M. Reingold, Graph drawing by force directed placement, Software: Practice and Experience 21(11) (1991), 1129-1164.

[11] G.W. Furnas, Generalized fisheye views, in: Proceedings of the SIGCHI Conference on Human Factors in Computing Systems, CHI'86, ACM, New York, NY, USA, 1986, pp. 16-23.

[12] J.B. Lovins, Development of a stemming algorithm, Mechanical Translation and Computational Linguistics 11 (1968), 22-31.

[13] J.B. Macqueen, Some methods for classification and analysis of multivariate observations, in: Proceedings of the 5th Berkeley Symposium on Mathematics, Statistics, and Probability, Vol. 1, UC Press, 1967, pp. 281-297.

[14] R. Mazza, Introduction to Information Visualization, Springer-Verlag, London, 2009.

[15] D. Nadeau and S. Sekine, A survey of named entity recognition and classification, Lingvisticae Investigationes 30(1) (2007), 3-26.

[16] C.D. Paice, Another stemmer, SIGIR Forum 24(3) (1990), 56-61.

[17] M.F. Porter, An algorithm for suffix stripping, Program 3(14) (1980), 130-137.

[18] A. Ratnaparkhi, A maximum entropy model for part-of-speech tagging, in: Proceedings of the Empirical Methods in Natural Language Processing, E. Brill and K. Church, eds, 1996, pp. 133-142.

[19] J. Rekimoto and M. Green, The information cube: Using transparency in 3d information visualization, in: Proceedings of the Third Annual Workshop on Information Technologies Systems, WITS'93, 1993, pp. 125-132.

[20] J.S. Risch, D.B. Rex, S.T. Dowson, T.B. Walters, R.A. May and B.D. Moon, The starlight information visualization system, in: Proceedings of the IEEE Conference on Information Visualisation, IV'97, IEEE Comp. Soc., Washington, DC, USA, 1997, pp. 42-49. 
[21] G.G. Robertson, J.D. Mackinlay and S.K. Card, Cone trees: animated 3d visualizations of hierarchical information, in: Proceedings of the SIGCHI Conference on Human Factors in Computing Systems: Reaching Through Technology, CHI'91, ACM, 1991, pp. 189-194.

[22] G. Salton, A. Wong and C.S. Yang, A vector space model for automatic indexing, Communications of the ACM 18(11) (1975), 613-620.

[23] H. Schmid, Probabilistic part-of-speech tagging using decision trees, in: Proceedings of the International Conference on New Methods in Language Processing, 1994, pp. 44-49.

[24] P. Shirley and S. Marschner, Fundamentals of Computer Graphics, 3rd edn, A.K. Peters, Ltd., Natick, MA, USA, 2009.

[25] B. Shneiderman, The eyes have it: A task by data type taxonomy for information visualizations, in: Proceedings of the IEEE Symposium on Visual Languages, IEEE, 1996, pp. 336-343.

[26] G. Zhou and J. Su, Named entity recognition using an hmm-based chunk tagger, in: Proceedings of the 40th Annual Meeting of the Association for Computational Linguistics, ACL, Stroudsburg, PA, USA, 2002, pp. 473-480. 